\title{
The analysis of image fusion based on improved Brovery transform
}

\author{
CHEN Rong \\ Nanchang Normal University, Nanchang, 330032, China \\ xinsea@163.com
}

Keywords: image, fusion, improved algorithm, brovery transform

\begin{abstract}
Image fusion is multi-source channel to collect on the same target image through image processing to extract certain, respective channel information, and finally integrated into the same image for observation or further processing.This paper presents a method of image fusion based on improved Brovery transform, and analyzes the the index evaluation of the fused image.
\end{abstract}

\section{Introduction}

Fusion algorithm is often combined with average value,standard deviation, entropy, average gradient of image.Average gradient reflects the minute details of contrast and texture change features in the image, but also reflects the image clarity.Image fusion method can according to the need of comprehensive processing of multi source channel information, thus effectively improving the utilization rate of image information and the reliability of target detection and recognition. Its objective is to multi-band information of a single sensor or similar sensor provides information to be integrated to eliminate possible between multiple sensor information redundancy and contradictions, in order to enhance the transparency of information and the reliability of image, to form the target clear, complete and accurate information description.

Data fusion is that the acquisition of various sources of information, multimedia and multi-format information, comprehensive information to generate the complete, accurate, timely and effective process.Study on data fusion method is how to process and collaborative use of multi-source information, so that the different forms of information to complement each other, thus finally get to know about the same object or target more objective.Fusion results provided by the information is more comprehensive and less redundant and more practical than each independent sources of information.Remote sensing image fusion is a branch of the theory of data fusion in remote sensing application, it focuses on those redundancy in space or time of multi-source data, computation and processing according to certain rules or algorithms,to obtain more accurate and more rich information than any single data.The two pretreatment steps before image fusion,first it must need spatial registration between images. The most common method is as the feature matching control points by selecting the obvious physical points, resampling for the same spatial resolution,usually the spatial registration error between images is no more than one pixel.The second is the image matching, in order to make the fused image reduce the spectral distortion,panchromatic image histogram transforms for corresponding histogram of multi-spectral images.Finally carries on the transformation using the fusion algorithm. Finally, it is using image fusion algorithm to transform ${ }^{[1][5]}$.

\section{Brovery transform fusion}

Brovery transform method is called the color standard transform fusion,It is for the RGB image display of multi spectral color normalization,the high-resolution panchromatic image and their multiplication complete fusion.It is multi-spectral color normalization for RGB image display.Image fusion is completed by the high-resolution panchromatic image and its multiplication.Brovery transform method is simply color normalization of image,fusion image well retains the original image color information, color coordination,clear images. This method is used to fusion,simple operation and can obtain the sharpening image while maintaining the original image spectral information at the 
same time effect.Edge and contour of fusion image is clear,help to extract feature boundary,texture and ground feature information after bravery transform method ${ }^{[2]}$.

In order to get more satisfactory fusion result,be amended to transform formula as follows ${ }^{[3]}$.

$$
\begin{aligned}
& \mathrm{R}=\mathrm{r}_{1} \times(\mathrm{r} /(\mathrm{r}+\mathrm{g}+\mathrm{b})) \times \mathrm{I}_{2}^{\mathrm{r}} \\
& \mathrm{G}=\mathrm{g}_{1} \times(\mathrm{g} /(\mathrm{r}+\mathrm{g}+\mathrm{b})) \times \mathrm{I}^{\mathrm{g}}{ }_{2} \\
& \mathrm{~B}=\mathrm{b}_{1} \times(\mathrm{b} /(\mathrm{r}+\mathrm{g}+\mathrm{b})) \times \mathrm{I}^{\mathrm{b}}{ }_{2}
\end{aligned}
$$

In formula, $r_{1}, r_{2}, g_{1}, g_{2}, b_{1}, b_{2}$ is coefficient of transformation formula, $r$ is the red band multi spectral image,g is the green band multi spectral image, $b$ is the blue band multi spectral image,I is the brightness images with high spatial resolution.The improved brovery transform simplifies the transformation algorithm, can improve the relevance of the luminance component between the original multi spectral image and fused image by adjusting the transform coefficient correlation of images,so as to retain the original spectral information of multi spectral images ${ }^{[4]}$.

Because the brovery transform is that the luminance component of multi spectral image is simple substitution for the high resolution image of I component, so will cause the fusion image histogram is compressed,brightness and contrast is reduced.Because of the existence of multi spectral image and high resolution image brightness component is inconsistency problem,will replace both, although can improve the spatial resolution of the fusion image,but also the inevitable result of a certain degree of distortion spectrum.Figure 1 shows the effect of brovery transform fusion.

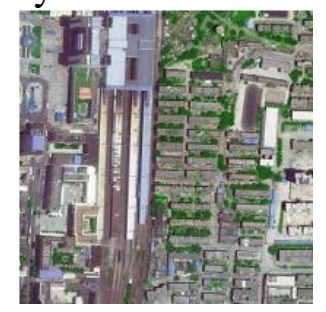

(a)spot1

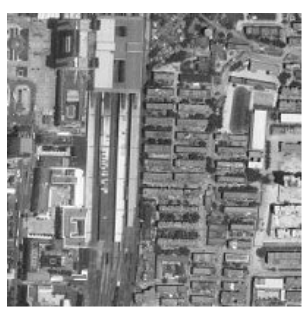

(b) spot2

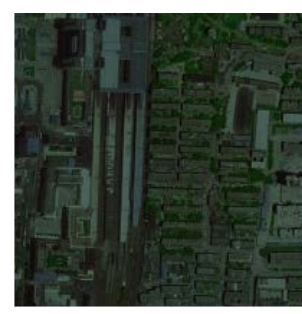

(c) fusion

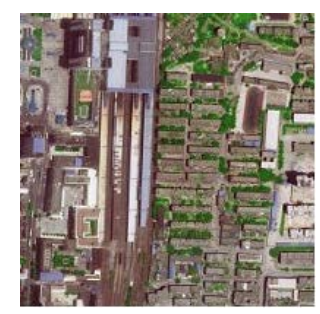

(d) Normalized fusion

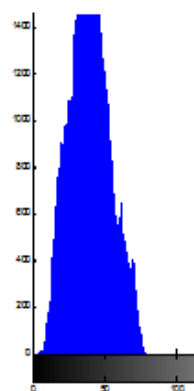

(e)fusion of R component

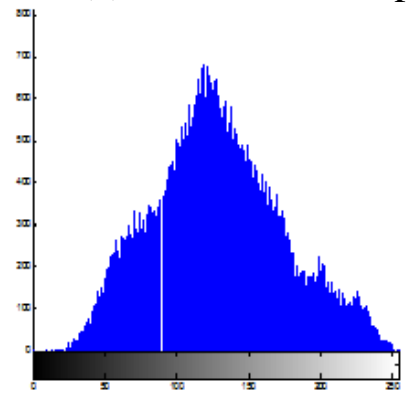

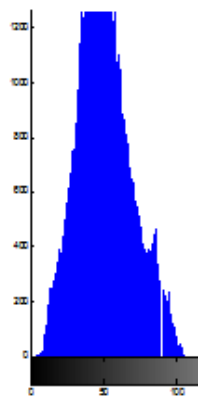

(f)fusion of G component

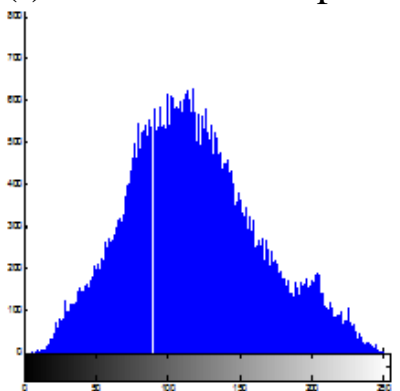

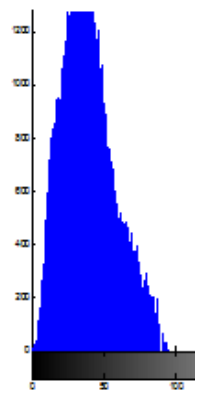

(g)fusion of B component

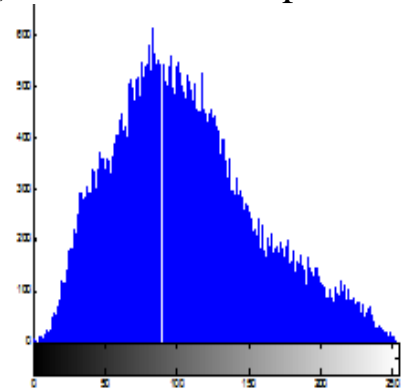

(h) normalized fusion of R component ( $i$ )normalized fusion of $G$ component $(j)$ normalized fusion of B component

Fig. 1 improved brovery fusion

\section{Fusion evaluation}

Evaluation method of fusion is the mean value,standard deviation,average error,measurement of information,correlation coefficient,contrast and peak signal to noise ratio.Mean value is the average gray value of all pixels in the image, which reflect the distribution of image gray. Close to 
the distribution of gray level images,the mean will be relatively close to.The standard deviation reflects the discrete image gray scale relative to the mean gray value,if the standard deviation is large,then the image gray level distribution of dispersion,image contrast is large,which can see more information. The small standard deviation, the image contrast is small,single color,do not see too much information.Image entropy is an important index to measure rich information of the image,if the entropy of fusion image is bigger, the amount of information increasing. If the entropy of fusion image is bigger,the more is the amount of fusion image information. Table 1 shows the index of fusion image.

Table 1 fusion image index

\begin{tabular}{cccc}
\hline & $\begin{array}{c}\text { Brovery } \\
\text { fusion }\end{array}$ & Brovery normalizedfusion & $\begin{array}{c}\text { Improved brovery } \\
\text { normalizedfusion }\end{array}$ \\
\hline mean value & 43.7600 & 126.1918 & 117.4097 \\
standard deviation & 15.6217 & 45.8861 & 47.9542 \\
entropy & 5.9574 & 7.5032 & 7.5032 \\
correlation & 0.9851 & 0.9833 & 0.9706 \\
coefficient & & 0.96 & \\
\hline
\end{tabular}

Note: $\mathrm{r}_{1}=0.9, \mathrm{r}_{2}=1.0 ; \quad \mathrm{g}_{1}=1.2, \mathrm{~g}_{2}=1.1 ; \quad \mathrm{b}_{1}=1.1, \mathrm{~b}_{2}=1.3$;

\section{Conclusion}

This paper introduces the method of the brovery transform in image fusion, and analyzed the evaluation of image fusion.It is the most important the evaluation of fusion image by improved brovery transform. The effect of fusion image by improved brovery transform is better than by brovery transform, and very simple adjustment coefficient of brovery, to achieve the best results fusion image.

\section{Fund Project}

Project supported by the key discipline of Nanchang Normal University,China (Grant No.NSXK20141001). At the same time, Project supported by the Reform in Education of Nanchang Normal University,China (Grant No.JGKT-14-18).

\section{Reference}

[1] LIU Zhe,HAO Chong-yang,FENG Wei,LIU Xiao-xiang,FAN Yang-yu. A Remotely Sensed Image Fusion Method Based on Wavelet Coefficient Features[J].Acta Geodaetica et Cartographica Sinica, 2004,33(1):53-57.

[2] SUN Rong-hua,GUO De-fang.Study on Fusion Algorithms of SPOT-5 PAN and Multi-spectral Images[J].Remote Sensing Technology And Application, 2005, 2005,20(3):366-370.

[3] LIU Zhe,HAO Chong-yang,LIU Xiao-xiang,FAN Yang-yu.Pixel-Based Image Fusion between PAN Image and Multispectral Image[J].Journal of Data Acquisition\&Processing,2003, 18(3):296-301.

[4] HUANG Yong-jie, WANG Shu-guo,CHEN Dong.Spectral preservation image fusion algorithm based on edge enhancement[J].Computer Engineering and Design,2009,30 (17): 4031-4034.

[5] Ricchetti E.Visible-infrared and radar i magery fusionfor geological application:a newapproach using DEMand sun-illumi-nation model. International Journal of Remote Sensing . 2001 